УДК 551.510:630*561.24

\title{
ТРЕНДЫ КЛИМАТИЧЕСКИХ ИЗМЕНЕНИЙ В ПЛОТНОСТИ ГОДИЧНЫХ КОЛЕЦ
}

\author{
Бондаренко Светлана Леонидовна', \\ bond sl@mail.ru
}

\author{
Устинова Ирина Георгиевна², \\ igu@tpu.ru \\ 1 Институт мониторинга климатических и экологических систем Сибирского отделения Российской академии наук, \\ Россия, 634055, г. Томск, пр. Академический, 10/3. \\ 2 Национальный исследовательский Томский политехнический университет, \\ Россия, 634050, г. Томск, пр. Ленина, 30.
}

\begin{abstract}
Актуальность исследования обусловлена необходимостью сохранности лесных зон и развития лесной индустрии в целом. Цель: использование многолетних трендов исследуемых характеристик: плотности годичных колец, изменений общего содержания озона в атмосфере и индекса аридности де Мортона для оценки климатических изменений; разложение дендрохронологического сигнала на компоненты, связанные с отдельными факторами.

объекты: временные ряды общего содержания озона в атмосфере, плотность годичных колец и индекс аридности де Мортона. Методы: анализ временных рядов, спектральный сингулярный анализ (метод «Гусеница», F-критерий).

Результаты. Был выполнен анализ дендрохронологических и климатических данных в двух численных экспериментах (Data Міning). Это позволило получить информацию для прогноза условий годичного прироста хвойных и связать отдельные компоненты дендрохронологического сигнала с воздействием определенных факторов (температура, осадки, ультрафиолет-В радиация или УФ-В и др.). Использование метода «Гусеница» в сочетании с предварительно выполненным факторным анализом дисперсии дендрохронологических рядов помогает выделить климатическую и УФ-В чувствительную (радиационную) компоненты в отдельные временные ряды. Мы можем использовать полученные компоненты для долгосрочного прогнозирования плотности древесины. Сигналы УФ-В радиации (общего содержания озона) и климатический - для реконструкции и прогноза атмосферных характеристик (общего содержания озона, индекса аридности де Мортона). Уравнения нелинейных трендов максимальной плотности годичных колец, общего содержания озона, индекса аридности де Мортона также позволяют получить достоверный прогноз условий формирования годичных колец и плотности древесины, но на меньший период, так как в них не учитывается цикличность климатических процессов.
\end{abstract}

\section{Ключевые слова:}

Временной ряд, тренд, прогнозирование, дендрохронологический метод, сингулярный спектральный анализ.

\section{Введение}

Засушливость и повышение среднегодовых температур окружающей среды, снижение уровня стратосферного озона с последующим повышением ультрафиолет-В (УФ-В) радиации в разной степени влияют на рост деревьев и состояние лесов. Прогнозирование и оценка условий формирования стволовой древесины хвойных деревьев могут быть полезны для сохранности лесных зон и развития лесной индустрии $[1,2]$.

Климат можно оценивать по индексу аридности де Мортона (ИМ) [3]. Этот параметр учитывает одновременно влияние влаги и тепла на рост деревьев. Таким образом, мы можем сократить число исследуемых факторов, влияющих на формирование древесины. Сеть станций наблюдений радиационного режима атмосферы - общего содержания озона (OCO) и УФ-В радиации, малочисленна, а временные ряды непродолжительны. Поэтому для продления (реконструкции) временных рядов в прошлое используются дендрохронологический метод и дендрохронологические характеристики, а именно максимальная плотность годичных колец [4].

Изменения природных факторов проявляются в трендах, амплитуде и периодах колебаний [5]. Трендом называют неслучайную функцию, кото- рая формируется под действием общих или долговременных тенденций, влияющих на временной ряд. Кроме того, существует понятие о тренде временного ряда плотности годичных колец как о возрастной кривой, связанной с особенностями отклика деревьев на изменения условий роста в зависимости от стадии развития [6]. Поэтому в представлении о трендах в климатических исследованиях необходимо отличать тренд от тенденции временного ряда. Тренд - это частный случай тенденции. Под трендом понимают основную закономерность в развитии случайного процесса (математическое ожидание случайного процесса). В отличие от тренда, тенденция ряда может образовывать циклы. Нередко в качестве основной тенденции временного ряда принимают его долгопериодную изменчивость [7].

Временные ряды, характеризующие радиационный $(\mathrm{OCO})$ и гидротермический (индекс аридности де Мортона ИМ) режимы содержат многолетние тренды. Проблема заключается в том, чтобы разделить влияние факторов в общем отклике (дендрохронологическом сигнале), выделить отдельно временные ряды, соответствующие откликам деревьев на воздействие УФ-В радиации и откликам на изменения ИМ. Применение метода 
спектрального сингулярного анализа (CatepillarSSA или «Гусеница») позволяет решить эту проблему. Несмотря на то, что в России (Санкт-Петербург) метод Caterpillar-SSA разработан довольно давно, его применение все еще находится в стадии освоения, хотя и позволяет получить оригинальные результаты [8-13].

Плотность годичных колец, основная характеристика структуры древесины, используется в дендрохронологических и климатологических исследованиях более 30 лет. $K$ сожалению, измерения плотности годичных колец - трудоемкий процесс, требующий дорогостоящей аппаратуры, в настоящее время базы данных новыми данными полноценно не пополняются.

Использование дендрохронологического метода для восполнения недостающих климатических данных сопряжено с определенными сложностями: сопоставление характеристик разных по своей природе процессов, различия по продолжительности, цикличности и чувствительности временных рядов.

Цель данной работы - оценка многолетних трендов исследуемых характеристик: плотности годичных колец (структура годичных колец), изменений общего содержания озона в атмосфере (влияние УФ-В радиации) и индекса аридности де Мортона (климатические условия).

\section{Методика}

Решение задачи идентификации параметров временного ряда [14] связано с поиском характеристики параметров реальной системы, породившей исследуемый временной ряд в прошлом и составлением прогноза поведения временного ряда в будущем $[15,16]$

Дендрохронологические временные ряды представляют собой усредненную максимальную плотность древесины кольца ствола $\mathrm{N}$ деревьев. Кольцо растет в течение теплого периода года, потом рост останавливается, за счет этого формируется визуально обнаруживаемая граница кольца, что и позволяет датировать изменения. Поэтому характеристики годичного кольца рассматриваются на временной шкале ряда среднемесячных или суммарных за теплый период значений исследуемых параметров. Существует большой набор дендрохронологических характеристик: ширина и плотность годичных колец, содержание микроэлементов или изотопов в годичных кольцах и др. Мы будем рассматривать максимальную плотность годичных колец (ПГК).

На ПГК действует множество факторов, отклики дерева на них обычно представляют в виде линейно-агрегативной модели [6]:

$$
\Pi \Gamma К(t)=A(t)+C(t)+D(t)+E(t),
$$

где $A(t)$ - тренд, вызванный физиологическими особенностями роста дерева; $C(t)$ - воздействие климатических факторов; $D(t)$ - другие природные воздействия; $(t)$ - случайная составляющая; $t$ - время.
Для прогноза условий роста деревьев желательно разделить дендрохронологический сигнал на отдельные составляющие. Такая делимость возможна при использовании методов анализа временных рядов, в частности сингулярного спектрального анализа. Метод позволяет разложить на компоненты даже слабо разделимые ряды. В основе метода лежит идея создания псевдоповторяемости временного ряда за счет смещения исходного ряда относительного него самого. Особенность использования метода Caterpillar-SSA состоит в том, что он позволяет анализировать данные временного ряда, не зная заранее периодичности и аналитической формы тренда этого ряда [17].

Временной ряд ПГК можно представить в виде тренда и суммы главных компонент, содержащих отклик дерева на изменения ОСО в атмосфере, интегральный отклик на климатический сигнал (индекс аридности де Мортона, характеризующий соотношение температуры и осадков) и остаточной компоненты, характеризующей отклики дерева на другие факторы (например, состояние почвы или продолжительность жизни хвои). Тренд формируется под действием общих многолетних тенденций, влияющих на ПГК природных факторов, и также может содержать низкочастотную составляющую.

В последнее время в научных исследованиях, в том числе связанных с изучением климата по дендрохронологическим данным, используются технологии Data Mining [18]. Такой подход позволяет ориентироваться в многочисленных этапах предварительной обработки данных и анализа.

В основе интеллектуального анализа (Data Mining) лежит предположение, что в дендрохронологическом сигнале содержится информация (временные ряды) о воздействии различных факторов на годичный прирост и что эту информацию можно ранжировать по степени корреляции с выбранным фактором и проценту объясненной дисперсии дендрохронологических данных. На рис. 1 приведена схема, из которой видно, что результатом DataMining является создание прогнозных моделей временных рядов.

\section{Тренды временного ряда}

Статистический прогноз на основе систематической составляющей временного ряда основан на экстраполяции, то есть предполагается, что параметры модели тренда сохраняются до прогнозируемого момента времени. Такую модель можно применять только к однородным процессам, числовые значения которых определяются постоянным набором причин [19]. Изменения ПГК, ОСО, температуры и осадков можно отнести к однородным временным рядам.

Приведем пример долгосрочного прогноза.

Пусть имеется временной ряд $y(t)=\varphi(t)+\xi(t)$, где $\varphi(t)$ - тренд временного ряда, $\xi(t)$ - случайная составляющая, $M\left[\xi\left(t_{i}\right)\right]=0, i=\overline{1, N}, D\left[\xi\left(t_{i}\right)\right]=\sigma^{2}$. Последовательность значений $y\left(t_{1}\right), y\left(t_{2}\right), \ldots, y\left(t_{N}\right)$ нам 
Сезонные (вегетационный период) и определенного рода высокочастотные колебания временных рядов (фильтрация, сглаживание)

Seasonal (vegetation period) and certain kind of high-frequency oscillations of time series (filtration, smoothing)

Связи между изменениями окружающей среды и характеристиками годичных колец (корреляционный анализ)

Relationship between environmental changes and the characteristics of annual rings (correlation analysis)

Опредение групп деревьев по чувствительности к отдельным видам изменений условий среды (кластерный анализ)

Determination of tree groups by sensitivity to individual types

of environmental conditions changes (cluster analysis)

Главные факторы, влияющие на характеристики годичных колец в группах (анализ дисперсии временных рядов)

Pricipal factors effecting the characteristics of annual rings in groups (time series analysis of variance)

Выделение компонент дендрохронологического сигнала, характеризующих отклики Decompose of components of the dendrochronological signal characterizing the response of trees to environmental changes (Caterpillar-SSA)

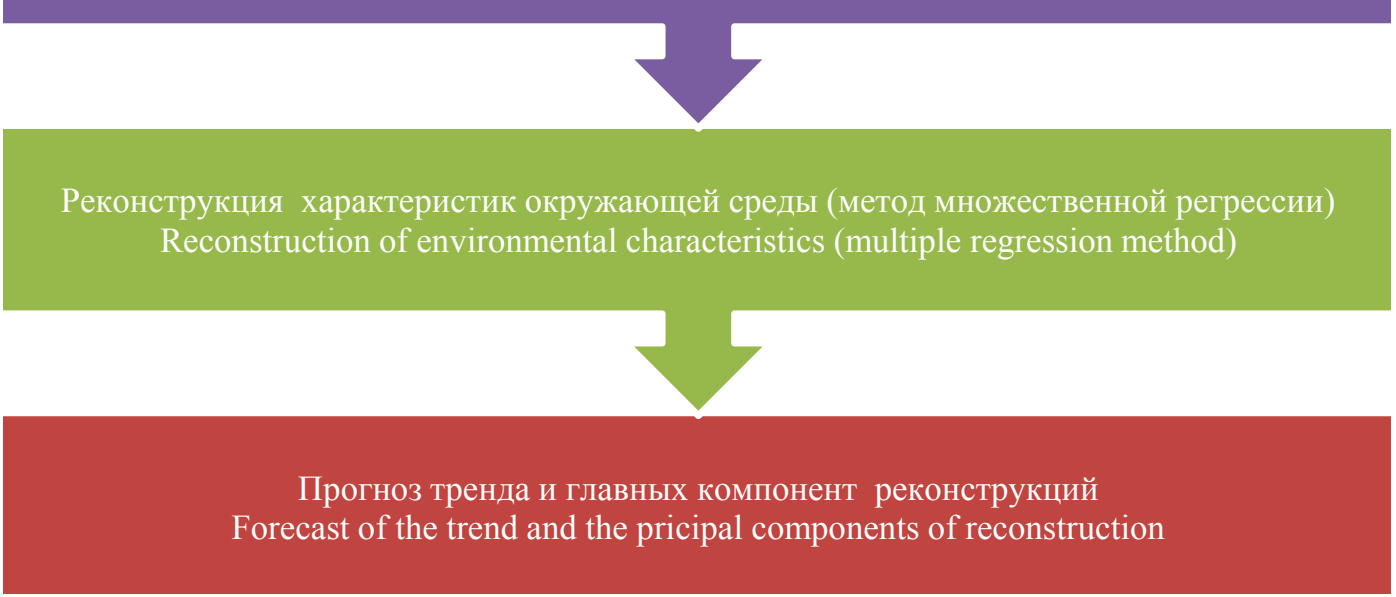

Pис.1. Блок-схема интеллектуального анализа (Data Mining)

Fig. 1. Block diagram of Data Mining

известна. Задача состоит в выделении тренда $\varphi(t)$ временного ряда $y(t)$.

Для решения задачи необходимо выбрать вид функции $\varphi(t)$. Чаще всего на практике используются следующие функции:

- линейная $\varphi(t)=a_{0}+a_{1} t$;

- полиномиальная $\varphi(t)=a_{0}+a_{1} t+a_{2} t^{2}+\ldots a_{n} t^{n}$;
- экспоненциальная $\varphi(t)=e^{a_{0}+a_{1} t}$;

- степенная $\varphi(t)=a_{0} t^{a_{1}}$.

Для выделения тренда используем метод наименьших квадратов, исходя из условия:

$$
Q=\sum_{i=1}^{N}\left[y_{i}-\varphi\left(t_{i}\right)\right]^{2} \Rightarrow \min _{a_{j}},
$$


где $j=\overline{0,1}$ в линейной, экспоненциальной и степенной модели тренда и $j=\overline{0, n}$ в полиномиальной модели тренда. Продифференцировав (1) по $a_{j}$, получаем систему нормальных уравнений, из которой ищутся параметры тренда.

Для оценки точности подобранной трендовой модели используется стандартный коэффициент детерминации [20]:

$$
R^{2}=\delta_{\hat{y}}^{2} / \delta_{y}^{2}
$$

где $\delta_{\hat{y}}^{2}=\sum_{i=1}^{N}\left(\hat{y}_{i}-\bar{y}\right)^{2} / N-$ дисперсия теоретических данных, полученных по трендовой модели; $\delta_{y}^{2}=\sum_{i=1}^{N}\left(y_{i}-\bar{y}\right)^{2} / N-$ дисперсия эмпирических данных.

\section{Трендовая компонента временного ряда}

Сингулярный спектральный анализ Caterpillar-SSA можно применять при исследовании стационарных и циклических рядов. Временные ряды ПГК, ОСО, температуры и осадков можно отнести и к тем и другим.

Основой метода Caterpillar-SSA является преобразование одномерного временного ряда в матрицу. Матрица формируется последовательным сдвигом рядов в 1 лаг. Чем длиннее серия, тем больше размер матрицы. Чем длиннее серия, тем больше число компонентов в вариантах разложения и сравнения. Программа «Caterpillar» обеспечивает визуализацию: принятия решений для факторного анализа, корреляционных матриц, периодограмм серий и компонентов, декомпозиции и суммирования компонентов, векторного и рекурсивного прогнозирования [21, 22]. Используя Саterpillar-SSA, временные ряды ПГК можно представить суммой колебаний различной периодичности и амплитуды. Основные компоненты разложения анализируются и затем суммируются в комбинации, определенной по результатам факторного анализа. Первая компонента временного ряда индексов, как правило, представляет собой долгопериодный тренд (если тренд не был удален на этапе подготовки данных).

Если используются абсолютные значения характеристик, то одна компонента составляет $99 \%$ от всего ряда. Возможно, это связано с отсутствием сильной разделимости компонент временного ряда в абсолютных значениях, вызванной близкими значениями собственных чисел компонент [23]. Поэтому используются стандартизованные ряды [24].

\section{Результаты}

Рассмотрим результаты численных экспериментов, приведенные на рис. 2-4.

Период пересечения данных всех временных рядов 1-го численного эксперимента - с 1932 по 2007 гг. Характеристика периода - значимые тренды в данных, характеризующих ИМ и УФ-В (OCO). Рассматривалась группа чувствительных к УФ-В временных рядов ПГК.

Период пересечения данных всех временных рядов 2-го численного эксперимента - с 1932 по 1974 гг. Характеристика периода - отсутствие значимых трендов в данных, характеризующих ИМ и УФ-В (ОСО). Рассматривалась группа чувствительных к УФ-В (ОСО) и климатическим изменениям (ИМ) временных рядов ПГК.

\section{Временной ряд ПГК}

Рассматривается временной ряд, представляющий собой региональную хронологию изменений максимальной плотности годичных колец для территории Альпийского хребта Франции и Швейцарии. Для получения продолжительного ряда усреднялись данные 160 серий ПГК деревьев, чувствительных к воздействию УФ-В радиации; из серий удалялись многолетние линейные тренды; в анализе использовались индексы, нормированные относительно среднего значения ряда.

Истинное значение плотности древесины кольца в 2007 г. равно 0,03699, тогда как значение плотности по представленной модели равно 0,03674. Разность между истинным значением параметра и расчетным составляет 0,00025 .

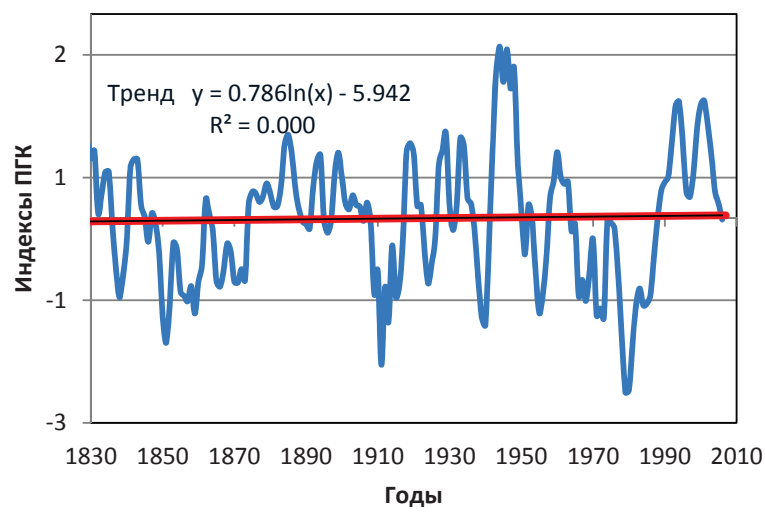

Puc. 2. Временной ряд с 1826 по 2007 г2. нормированной на среднее плотности годичных колеи (синял линия) и его логарифмическая аппроксимация (красная линия)

Fig. 2. Time series from 1826 to 2007 which was normed on the mean value of the density of annual rings (blue line) and its logarithmic approximation (red line)

Проверим значимость полученного уравнения тренда по F-критерию [25] на пятипроцентном уровне значимости. Вычисляем необходимые суммы квадратов по формулам:

- общая сумма квадратов

$$
Q=\sum_{i=1}^{N} y_{i}^{2}-\frac{\left(\sum_{i=1}^{N} y_{i}\right)^{2}}{N}=116,5332 ;
$$

- остаточная сумма квадратов

$$
Q_{e}=\sum_{i=1}^{N}\left(y\left(t_{i}\right)-\bar{y}\right)^{2}=0,081912,
$$


где $\bar{y}=\frac{\sum_{i=1}^{N} y_{i}}{N}-$ выборочное среднее.

Тогда

$$
Q_{R}=Q-Q_{e}=116,45129
$$

и

$$
F=\frac{Q_{R}(N-m)}{Q_{e}(m-1)}=\frac{116,45129(181-2)}{0,081912}>F_{0.05 ; 1 ; 179}=3,84
$$

( $m$ - число оцениваемых параметров).

В рассматриваемом выражении $m=2$, и, следовательно, полученное уравнение значимо.

Общее содержание озона в единицах Добсона (е.Д.) в 2011 г. равно 319,728 е.Д. Общее содержание озона в соответствии с полученной моделью тренда равно 320,941 е.Д. Разность между истинным значением и оценкой равна 1,214 е.Д. Общая сумма квадратов

$$
Q=\sum_{i=1}^{N} y_{i}^{2}-\frac{\left(\sum_{i=1}^{N} y_{i}\right)^{2}}{N}=5155,028 \text { е.Д., }
$$

остаточная сумма квадратов

$$
Q_{e}=\sum_{i=1}^{N}\left(y\left(t_{i}\right)-\bar{y}\right)^{2}=4397,238 \text { е.Д. }
$$

Тогда

$$
\begin{gathered}
Q_{R}=Q-Q_{e}=757,790 \text { e.Д. } \\
F=\frac{Q_{R}(N-m)}{Q_{e}(m-1)}=\frac{757,7903(79-2)}{4397,238}=13,27>F_{0.05 ; ; ; 79},
\end{gathered}
$$

следовательно, полученное уравнение значимо.

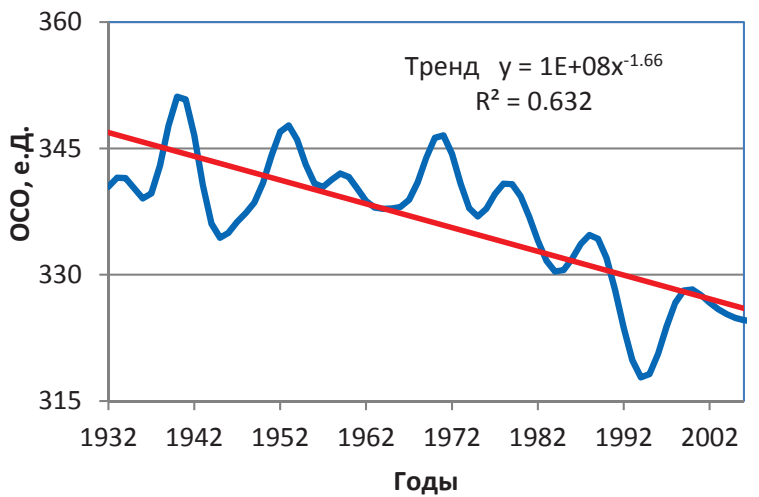

Рис. 3. Временной ряд с 1932 по 2007 г2. общего содержания озона (синяя линия) и его степенная аппроксимация (красная линия)

Fig. 3. Time series from 1932 to 2007 of total ozone (blue line) and its power-law approximation (red line)

\section{Временной ряд ИМ}

Временной ряд ИМ отражает одновременное воздействие на дендрохронологические данные двух факторов: температуры и влажности. Значения ИМ приводятся в мм/град, также могут использоваться нормированные (безразмерные) индексы ИМ.

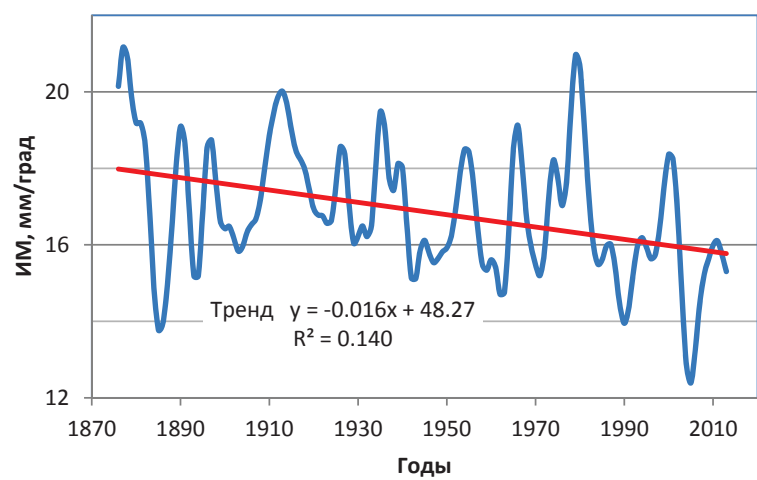

Pис.4. Временной ряд (1876-2006 г2.) индексов аридности де Мортона (синяя линия) и его тренд. Линейная аппроксимация представлена красной линией

Fig. 4. Time series (1876-2006) of the De Martonne aridity index (blue line) and its trend. Linear approximation is represented by the red line

Индекс аридности лучше всего описывается с помощью экспоненциального тренда. Истинное значение индекса аридности на 2014 г. равно 15,3455 мм/град, найденное по экспоненциальному тренду равно 14,5344 мм/град. Разность составляет 0,8110 мм/град. Общая сумма квадратов

$$
Q=\sum_{i=1}^{N} y_{i}^{2}-\frac{\left(\sum_{i=1}^{N} y_{i}\right)^{2}}{N}=394,6728 \text { мм/град, }
$$

остаточная сумма квадратов

$$
Q_{e}=\sum_{i=1}^{N}\left(y\left(t_{i}\right)-\bar{y}\right)^{2}=280,5727 \text { мм/град. }
$$

Тогда

$$
\begin{gathered}
Q_{R}=Q-Q_{e}=114,1001 \mathrm{мм} / \text { град. } \\
F=\frac{Q_{R}(N-m)}{Q_{e}(m-1)}=\frac{114,1001(138-2)}{280,5727}= \\
=55,307>F_{0.05 ; ; 1 ; 36},
\end{gathered}
$$

следовательно, полученное уравнение значимо. Для второго численного эксперимента (рис. 5) использовались данные 160 серий ПГК для территории Швейцарии. Данные были разделены на две группы: чувствительные к УФ-В и чувствительные к изменениям климата.

Согласно таблице, на рис. 5 представлены долгопериодные тренды и главные компоненты усредненных по группам 1 (а) и 2 (б) рядов ПГК. Разложение на тренд и главные компоненты выполнялось по 50 компонентам (половина численности рядов ПГК) в программе Caterpillar. В группе деревьев, чувствительных к УФ-В (ОСО) воздействию, первая компонента представляет собой долгопериодный тренд и составляет $18 \%$, УФ-В сигнал - $38 \%$ $(2-4,19$ компоненты разложения) усредненного по группе ПГК ряда. Для группы деревьев, чувствительных к изменениям климата, первая компонента (тренд) составляет $17 \%$, а климатический сигнал - 61 \% (2-8, 19 компоненты усредненного по группе ряда). 


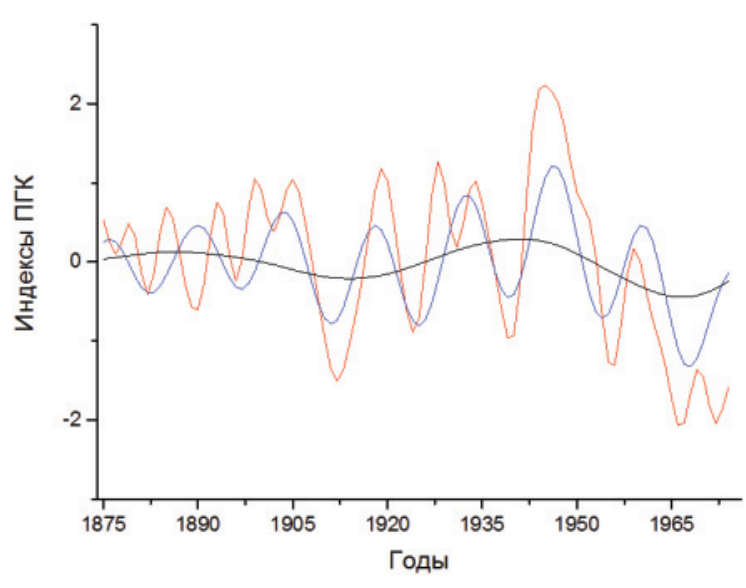

$a / a$

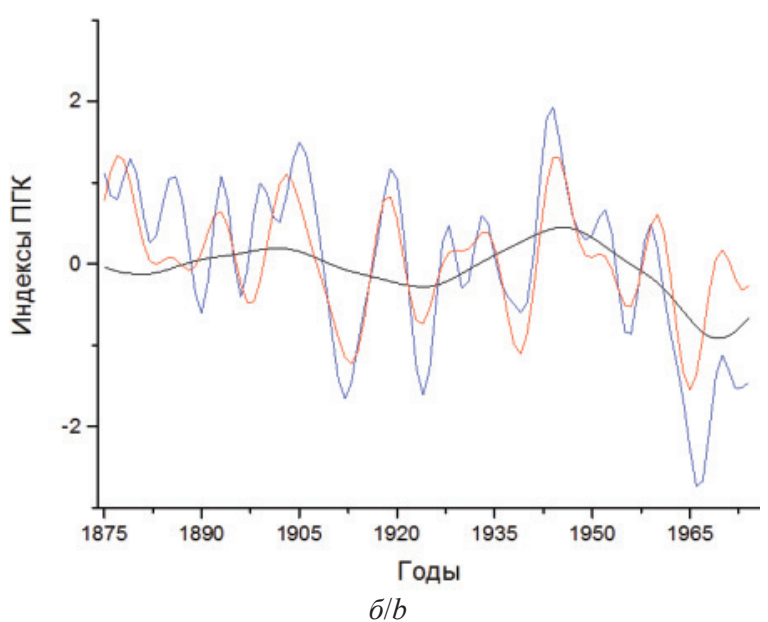

таблица. Р Распределение главных компонент в группе столет них рядов хронологий плотности. Колонка с данными по первой группе представляет главные колпо ненты чувствительных к УФ-В деревьев, колонка с данныли по второй группе - чувствительных к климатическим изленениям деревьев. Дисперсия откли ков на изленение УФ-В оценивалась по данныл ОСО. Сумларный процент общей дисперсии откликов ПГК деревьев на изменения ИМ и ОСО в обеих груп пах составляет $70 \%$

Table. Distribution of main components in the group of centenary series of density chronologies. The column with data of the group 1 represents the main components of trees, sensitive to the UV-B, the column with the data of the group 2 represents the main components of trees, sensitive to climatic changes. The dispersion of responses to the change in UV-B was estimated by the data of the total ozone content (TOC). The cumulative percentages of general dispersion to changes the De Martonne aridity index (IDM) and TOC in both groups is $70 \%$

\begin{tabular}{|c|c|c|}
\hline \multirow{2}{*}{ Фактор } & \% общей дисперсии \% /general variance \\
\cline { 2 - 3 } Factor & \multicolumn{2}{|c|}{ группа/group } \\
\cline { 2 - 3 } & 1 & 2 \\
\hline \multirow{2}{*}{1} & 38,03 (ОСО) & 60,7 (ИМ) \\
& 38,03 (ТОС) & 60,7 (IDM) \\
\hline \multirow{2}{*}{2} & 26,97 (ИМ) & $8,5($ OCO) \\
& 26,97 (IDM) & 8,5 (ТОС) \\
\hline
\end{tabular}

Puc.5. Разложение методом Caterpillar-SSA временных рядов нормированной на среднее плотности годичных колеи, чувствительных к: а) УФ-В воздействию (ОСО); б) климатическим изленениям ИМ, на колпоненты согласно таблице: дендрохронологические сигналы в ПГК чувствительные к УФ-В радиации - красная линия, к клилату синяя линия, долгопериодные тендениии - черная линия

Fig. 5. Decomposition (using the Caterpillar method) of time series normed on the mean value of the density of annual rings sensitive to: a) UV-B effects (TOC); b) climatic changes (IDM), into components according to the table: a dendrochronological signal in MXD (maximum tree-ring density) of responsive to $U V-B$ trees is shown by the red line; a climatic signal is shown by the blue line; long-term trends in the time series are shown by the black lines $(a, b)$

Рис. 5 показывает цикличность откликов деревьев на изменения экологических факторов различной природы. Цикличность в долгосрочных тенденциях связана с чередованием благоприятных и неблагоприятных условий роста деревьев.

Рис. 6 иллюстрирует верификацию разложения временного ряда ПГК, чувствительного к УФ-В фактору по данным временного ряда ПГК с 1826 по 2007 гг. Прогноз (верификация данных разложения) выполнен в программе CaterpillarSSA v. 3.40.

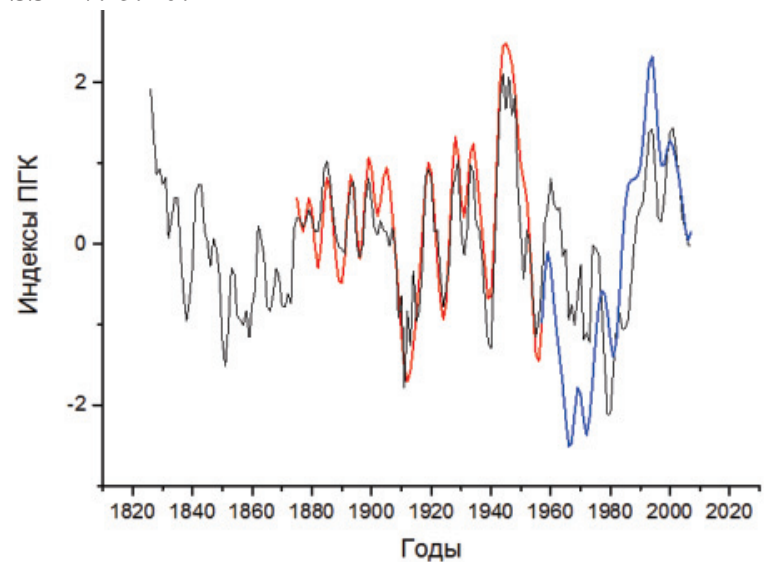

Puс.6. Верификаиия (синяя линия) временного ряда нормиро ванной на среднее плотности годичных колеи, чувстви тельных к УФ-В воздействию (красная линия), в сравнении с более продолжительным рядом ПГК с 1826 по 2007 г2. (черная линия)

Fig. 6. Verification (blue line) of the time series normalized to the mean value annual density of rings sensitive to UV-B effect (the red line) in comparison with a more longer time series of MXD from 1826 to 2007 (black line)

\section{Обсуждение результатов}

В дендрохронологии тренд характеристик годичных колец равнозначен понятию «возрастная кривая». Это означает, что свойства дерева и характеристики годичных колец изменяются с возрастом. Возрастную кривую принято удалять из данных. Особенно это важно для характеристики ширины годичного кольца, для ПГК - в меньшей степени. Для климатических параметров тренды содержат часть дозы воздействия УФ-В радиации 
или количество градусов снижения или повышения температуры и т. д. Таким образом, удаляя тренды из временного ряда мы уменьшаем или увеличиваем степень воздействия внешнего фактора. Нужно ли убирать тренды из временных рядов для реконструкции климатических параметров? Так, на этапе поиска корреляций предварительной обработки данных численного эксперимента 1 из временных рядов ПГК были удалены линейные тренды. В период с трендом изменений ОСО, близким к нулю, корреляция плотности годичных колец и общего содержания озона была умеренной. Далее, когда тенденция снижения общего содержания озона усилилась, мы ожидали, что корреляция между параметрами возрастет до высокой. Однако при удалении тренда из временных данных ПГК корреляция между ОСО и ПГК напротив практически отсутствовала. В этом случае можно предположить ошибку в алгоритме обработки данных. При сохранении трендов в данных ПГК предполагаемая закономерность сохранялась и усиливалась. Поэтому в численном эксперименте 1 тренды из временных рядов ОСО не удалялись.

Временные ряды исследуемых характеристик имеют нелинейные тренды, что соответствует сложившимся модельным представлениям [26, 27].

Временные ряды с откликами деревьев на изменения условий окружающей среды содержат тренды и главные компоненты, указывающие на циклическую природу климатических и радиационных процессов, а также на заметные временные сдвиги между ними.

\section{СПИСОК ЛИТЕРАТУРЫ}

1. Данилов Д.А. Влияние лесохозяйственных воздействий и состава насаждений на анатомические показатели и плотность древесины сосны и ели // Лесной вестник. - 2016. - № 4. C. $15-19$.

2. Данилов Д.А., Скупченко В.Б. Изменения в строении древесины сосны и ели на анатомическом уровне в древостоях, пройденных рубками ухода и комплексным уходом // Лесной журнал. - 2014. - № 5. - С. 70-88.

3. Projected and Observed Aridity and Climate Change in the East Coast of South India under RCP 4.5. / A. Ramachandran, D. Praveen, R. Jaganathan, K. Palanivelu // The Scientific World Journal. - 2015. URL: http://dx.doi.org/10.1155/2015/169761 (дата обращения 05.04.2018)

4. Зуев В.В., Бондаренко С.Л. Исследование озоносферы методами дендрохронологии. - Томск: Изд-во СОРАН, 2007. - 168 с.

5. Intra-annual density fluctuations in tree rings: how, when, where, and why? / V. de Micco, F. Campelo, M. de Luis, A. Bräuning, M. Grabner, G. Battipaglia, P. Cherubini // IAWA Journal. 2016. - V. 37. - № 2. - P. 232-259.

6. Тишин Д.В. Дендроэкология (методика древесно-кольцевого анализа). - Казань: Казан. ун-т, 2015. - 36 c. URL: http:// dspace.kpfu.ru/xmlui/bitstream/handle/net/21965/ 0282 000873.pdf (дата обращения 05.04.2018).

7. Kerry A.E. What we know about climate change. - Boston: MIT Press, 2012. -96 p.

8. Шишов В.В., Попкова М.И., Тычков И.И. Нелинейное оценивание дендроклиматических данных и его применение для территории Сибири. - Красноярск: 000 рПб «Амальгама», 2016. - 128 с.

\section{Заключение}

Интеллектуальный анализ дендрохронологических и климатических данных (Data Mining) позволяет получить информацию для прогноза условий годичного прироста хвойных и связать отдельные компоненты дендрохронологического сигнала с воздействием определенных факторов (температура, осадки, УФ-В радиация и др.). Использование метода Caterpillar-SSA в сочетании с предварительно выполненным факторным анализом дисперсии дендрохронологических рядов, рассмотренным во втором численном эксперименте, помогает выделить эти компоненты в отдельные временные ряды. Мы можем использовать полученные компоненты для долгосрочного прогнозирования плотности древесины, а сигналы УФ-В (ОСО) и климатический - для реконструкции и прогноза атмосферных характеристик (ОСО, ИМ). Уравнения трендов ПГК, ОСО, ИМ, рассмотренные в первом численном эксперименте, также позволяют получить достоверный прогноз условий формирования годичных колец и плотности древесины, но на меньший период, так как в них не учитывается цикличность климатических процессов.

Исследование было выполнено в рамках госбюджетной темы № AAАA-A17-117013050038-7. Разработанная методика позволяет прогнозировать изменения климатического и радиационного режимов атмосферы на территориях для которых имеются необходимые данные и лесная растительность, в том числе и для Сибири.

9. Bondarenko S.L., Savchuk, D.A. Response of conifers to UV-B and climate in mountain areas // Global. J. Environ. Sci. Manage. - 2018. - № 4 (3) - P. 299-314.

10. Golyandina N., Korobeynikov A., Zhigljavsky A. Singular Spectrum Analysis with R. - Berlin: Springer-Verlag Heidelberg, 2018. - $272 \mathrm{p}$

11. Long-term $\mathrm{H}_{2} \mathrm{O}$ and $\mathrm{CO}_{2}$ trends in conifer disc tree rings and meteorological parameters / B.G. Ageev, A.N. Gruzdev, S.L. Bondarenko, V.A. Sapozhnikova // Journal of Life Sciences. - 2013. № 7. - P. 1002-1008.

12. Polukoshko S., Hilkevica G., Gonca V. Nonstationary Processes Studying Based on «Caterpillar»-SSA // Proc. of the $11^{\text {th }}$ International Conference of Vibration Engineering and Technology of Machinery. - UK: University of Manchester, 2014. - V. 23. P. 1002-1008.

13. Hassani H., Heravi S., Zhigljavsky A. Forecasting UK Industrial Production with Multivariate Singular Spectrum Analysis // The Journal of Forecasting. - 2013. - V. 32. - P. 395-408.

14. Кремер Н.Ш. Теория вероятностей и математическая статистика. - М.: ЮНИТИ, 2007. -550 с.

15. Idrisov F.F., Konstantinova I.G. Identification of trends of time series for a random number of measurements // Russian Physics Journal. - 1999. - V. 42. - P. 379-384.

16. Anderson T.W. The Statistical Analysis of Time Series. - New York: John Wiley, 1971. $-751 \mathrm{p}$

17. Golyandina N.E., Usevich K.D. 2D-extension of Singular Spectrum Analysis: algorithm and elements of theory // Matrix Methods: Theory, Algorithms, Applications. - Singapore: World Scientific, 2010. - P. 449-473. 
18. Using Data Mining to Predict Soil Quality after Application of Biosolids in Agriculture / J. Cortet, D. Kocev, C. Ducobu, S. Džeroski, M. Debeljak, C. Schwartz // J. Environ. Qual. - 2011. V. 40. - № 6. - P. 1972-1982.

19. Fritts H.C. Tree rings and climate. - New York: The Blackburn Press, 2001. $-582 \mathrm{p}$.

20. Афанасьева Т.В., Наместников А.М., Перфильева И.Г., Романов А.А. Прогнозирование временных рядов: нечеткие модели. - Ульяновск: УлГТУ, 2014. -145 с.

21. Golyandina N., Zhigljavsky A. Singular Spectrum Analysis for Time Series. - Berlin; Heidelberg: Springer-Verlag, 2013. $120 \mathrm{p}$.

22. De Carvalho M., Rua A. Real-time now casting the US output gap: Singular spectrum analysis at work // International Journal of Forecasting. - 2017. - V. 33. - № 1. - C. 185-198.

23. Box G.E.P., Jenkins G.M. Time Series Analysis: Forecasting and Control. - San Francisco: Holden Day, 1970. - 406 p.
24. Бахрушин В.Е. Методы оценивания характеристик нелинейных статистических связей // Системные технологии. 2011. - T. 2. - № 73. - С. 9-14.

25. Зуев В.В. Биоиндикация стратосферного озона. - Новосибирск: Изд-во СО РАН, 2006. - 228 с.

26. Ross Sh.M. Introduction to probability and statistics for engineers and scientists. - San Diego: Harcourt Academic Press, 2000. $-578 \mathrm{p}$.

27. Параметры роста деревьев на основе модели Ваганова-Шашкина в Центральной Сибири / И.И. Тычков, М.И. Попкова, А.Н. Николаев, В.В. Шишов // Вестник Северо-Восточного федерального университета им. М.К. Аммосова. - 2015. № 6 (50). - C. 40-52.

\section{Информация об авторах}

Бондаренко C.J., кандидат физико-математических наук, старший научный сотрудник Института мониторинга климатических и экологических систем Сибирского отделения Российской академии наук.

Устинова И.Г., кандидат технических наук, доцент школы базовой инженерной подготовки Национального исследовательского Томского политехнического университета. 


\title{
TRENDS OF CLIMATIC CHANGES IN DENSITY OF YEAR RINGS
}

\author{
Svetlana L. Bondarenko', \\ bond_sl@mail.ru \\ Irina G. Ustinova² \\ igu@tpu.ru \\ 1 Institute of Monitoring of Climatic and Ecological Systems Siberian Branch of the Russian Academy of Sciences, \\ 10/3, Akademichesky Avenue, Tomsk, 634055, Russia. \\ 2 National Research Tomsk Polytechnic University, \\ 30, Lenin Avenue, Tomsk, 634050, Russia.
}

The relevance of the research is caused by the need to conserve forest zones and to develop forest industry.

The main aim of the research is to use long-term trends in characteristics under study such as density of annual rings, changes in total ozone in the atmosphere and the de Martonne aridity index for assessing climate change. In addition, another aim of the study is to decompose the dendrochronological signal into components associated with individual factors.

Objects of the research are time series of the total ozone content in the atmosphere, density of annual rings and the de Martonne aridity index.

Methods: time series analysis, spectral singular analysis (Caterpillar-SSA method), F-criterion.

Results. The authors have analyzed the dendrochronological and climatic data in two numerical experiments (Data Mining). This allowed obtaining the information for the forecast of annual growth of coniferous trees and linking the individual components of the dendrochronological signal with the influence of certain factors (temperature, precipitation, ultraviolet-B radiation, etc.). The use of the Caterpillar-SSA method in combination with previously made factor analysis of the dispersion of the dendrochronological series helps allocate climatic and sensitive to ultraviolet-B radiation components in separate time series. The obtained components can be used for longterm prediction of wood density. Ultraviolet-B radiation (total ozone content) and climate signal can be used for reconstruction and prediction of atmospheric characteristics (total ozone content, the de Martonne aridity index). Equations of nonlinear trends of maximum density of annual rings of coniferous trees, of total ozone content, of the de Martonne aridity index also allow obtaining a reliable prediction of the conditions of formation of annual rings and wood density, but for a shorter period, as they do not consider the cyclicity of climatic processes.

Key words:

Time series, trend, prediction, dendrochronological method, singular spectral analysis.

\section{REFERENCES}

1. Danilov D.A. The effect of silvicultural influences and forest stand structure of anatomic indicators and density of pine and spruce wood. Forestry Bulletin, 2016, no. 4, pp. 15-19. In Rus.

2. Danilov D.A., Skupchenko V.B. Changes in the Structure of Pine and Spruce on the Anatomical Level in the Stands Passed Cutting and Complex Care. Lesnoy zhurnal, 2014, no. 5, pp. 70-88. In Rus.

3. Ramachandran A., Praveen D., Jaganathan R., Palanivelu K. Projected and Observed Aridity and Climate Change in the East Coast of South India under RCP 4.5. The Scientific World Journal, 2015. Available at: http://dx.doi.org/10.1155/2015/ 169761 (accessed 5 April 2018).

4. Zuev V.V., Bondarenko S.L. Issledovanie ozonosfery metodami dendrokhronologii [Study of ozonosphere by dendrochronological method]. Tomsk, SORAN Publ., 2007. 168 p.

5. De Micco V., Campelo F., De Luis M., Bräuning A., Grabner M., Battipaglia G., Cherubini P. Intra-annual density fluctuations in tree rings: how, when, where, and why? IAWA Journal, 2016, vol. 37 , no. 2, pp. 232-259.

6. Tishin D.V. Dendroekologiya (metodika drevesno-koltsevogo ana liza ). Kazan, Kazan University Publ., 2011. 33 p. Available at: http://kpfu.ru/staff_files/F624353973/dendroecology.pdf (accessed 5 April 2018).

7. Kerry A.E. What we know about climate change. Boston, MIT Press, $2012.96 \mathrm{p}$

8. Shishov V.V., Popkova M.I., Tychkov I.I. Nelineynoe otsenivanie dendroklimaticheskikh dannykh i ego primenenie dlya territory Sibiri [Nonlinear estimation of dendroclimatic data and its appli- cation to the territory of Siberia]. Krasnoyarsk, Amalgama Publ., $2016.128 \mathrm{p}$.

9. Bondarenko S.L., Savchuk D.A. Response of conifers to UV-B and climate in mountain areas. Global. J.Environ. Sci. Manage, 2018, no. 4 (3), pp. 299-314.

10. Golyandina N., Korobeynikov A., Zhigljavsky A. Singular Spectrum Analysis with R. Berlin, Springer-Verlag Heidelberg, 2018. $272 \mathrm{p}$.

11. Ageev B.G., Gruzdev A.N., Bondarenko S.L., Sapozhnikova V.A. Long-term $\mathrm{H}_{2} \mathrm{O}$ and $\mathrm{CO}_{2}$ trends in conifer disc tree rings and meteorological parameters. Journal of Life Sciences, 2013, no. 7, pp. 1002-1008.

12. Polukoshko S., Hilkevica G., Gonca V. Nonstationary Processes Studying Based on «Caterpillar»-SSA. Proc. of the $11^{\text {th }}$ International Conference of Vibration Engineering and Technology of Machinery. UK, University of Manchester, 2014. Vol. 23, pp. 1002-1008.

13. Hassani H., Heravi S., Zhigljavsky A. Forecasting UK Industrial Production with Multivariate Singular Spectrum Analysis. The Journal of Forecasting, 2013, vol. 32, pp. 395-408.

14. Kremer N.Sh. Teoriya veroyatnostey i matematicheskaya statisti$k a$ [Probability theory and mathematical statistics]. Moscow, YUNITI Publ., 2007. $550 \mathrm{p}$.

15. Idrisov F.F., Konstantinova I.G. Identification of trends of time series for a random number of measurements. Russian Physics Journal, 1999, vol. 42, pp. 379-384.

16. Anderson T.W. The Statistical Analysis of Time Series. New York, John Wiley, 1971. $751 \mathrm{p}$.

17. Golyandina N.E., Usevich K.D. 2D-extension of Singular Spectrum Analysis: algorithm and elements of theory. Matrix 
Methods: Theory, Algorithms, Applications. Singapore, World Scientific, 2010. pp. 449-473.

18. Cortet J., Kocev D., Ducobu C., Džeroski S., Debeljak M., Schwartz C. Using Data Mining to Predict Soil Quality after Application of Biosolids in Agriculture. J. Environ. Qual., 2011, vol. 40, no. 6, pp. 1972-1982.

19. Fritts H.C. Tree rings and climate. New York, The Blackburn Press, 2001. $582 \mathrm{p}$.

20. Afanaseva T.V., Namestnikov A.M., Perfileva I.G., Romanov A.A. Prognozirovanie vremennykh ryadov: nechetkie modeli [Forecasting time series: fuzzy models]. Ulyanovsk, UlGTU Publ., $2014.145 \mathrm{p}$.

21. Golyandina N., Zhigljavsky A. Singular Spectrum Analysis for Time Series. Berlin, Heidelberg, Springer-Verlag, 2013.120 p.

22. De Carvalho M., Rua A. Real-time now casting the US output gap: Singular spectrum analysis at work. International Journal of $F_{0}$ recasting, 2017, vol. 33, no. 1, pp. 185-198.
23. Box G.E.P., Jenkins G.M. Time Series Analysis: Forecasting and Control. San Francisco, Holden Day, 1970. 406 p.

24. Bahrushin V.E. Metody otsenivaniya kharakteristik nelineynykh statisticheskikh svyazey [Methods for estimating characteristcs of non-linear statistic bounds]. Sistemnye tekhnologii, 2011, vol. 2, no. 73, pp. 9-14.

25. Zuev V.V. Bioindikatsiya stratosfernogo ozona [Bioindexation of stratosphere ozone]. Novosibirsk, SB RAN Publ., 2006. 228 p.

26. Ross Sh.M. Introduction to probability and statistics for engineers and scientists. San Diego, Harcourt Academic Press, 2000. $578 \mathrm{p}$.

27. Tychkov I.I., Popkova M.I., Nikolaev A.N., Shishov V.V. Parameters of Tree Growth Based on Vaganov-Shashkin Model in Central Siberia. Vestnik Severo-Vostochnogo federalnogo universiteta im.M.K.Ammosova, 2015, no. 6 (50), pp. 40-52. In Rus.

Received: 18 October 2018.

\section{Information about the authors}

Svetlana L. Bondarenko, Cand. Sc., senior researcher, Institute of Monitoring of Climatic and Ecological Systems Siberian Branch of the Russian Academy of Sciences.

Irina G. Ustinova, Cand. Sc., associate professor, National Research Tomsk Polytechnic University. 